Supplement of J. Micropalaeontol., 40, 101-144, 2021

https://doi.org/10.5194/jm-40-101-2021-supplement

(C) Author(s) 2021. CC BY 4.0 License.

Journal of

(c) (1)

Micropalaeontology

Supplement of

\title{
Revised taxonomy and early evolution of fasciculiths at the Danian-Selandian transition
}

\section{Francesco Miniati et al.}

Correspondence to: Francesco Miniati (francesco.miniati@unimi.it)

The copyright of individual parts of the supplement might differ from the article licence. 
Table S1. Species, specimens, site/section and inventory number of all the specimens investigated in this study. Type materials and images for this study are stored at the Geological and Paleontological Museum (IGF code) and the Department of Earth Sciences of the University of Florence (DST code). Address: Via Giorgio La Pira 4, 50121 Firenze, Italy.

\begin{tabular}{|c|c|c|c|c|c|}
\hline \multirow[t]{2}{*}{ Species } & \multirow[t]{2}{*}{ Specimen } & \begin{tabular}{|l|} 
Inventory \\
Number \\
\end{tabular} & \multirow[t]{2}{*}{ Site/Section/Sample } & \multirow{2}{*}{$\begin{array}{l}\text { Inventory } \\
\text { Number } \\
\text { Sample } N^{\circ}\end{array}$} & \\
\hline & & Specimen $\mathbf{N}^{\circ}$ & & & \\
\hline \multirow[t]{12}{*}{ Gomphiolithus magnus } & Fig $08(1)$ & IGF 104803 & 1209B 25-1, 142-143 & IGF 104803-1 & STAB 12 \\
\hline & Fig $08(2)$ & IGF 104804 & 1209B 25-1, 142-143 & IGF 104803-1 & STAB 12 \\
\hline & Fig $08(3)$ & IGF 104805 & 1209B 25-1, 142-143 & IGF 104803-1 & STAB 12 \\
\hline & Fig $08(4)$ & IGF 104806 & Contessa Highway $10.79 \mathrm{~m}$ & IGF 104806-1 & Smear slide \\
\hline & Fig $08(5)$ & IGF 104807 & Bottaccione $10.60 \mathrm{~m}$ & IGF 104807-1 & Smear slide \\
\hline & Fig $08(6)$ & IGF 104808 & Bottaccione $11.38 \mathrm{~m}$ & IGF 104808-1 & Smear slide \\
\hline & Fig $08(7)$ & IGF 104809 & $1262 \mathrm{C} 11-5,131-132$ & IGF 104809-1 & $\begin{array}{l}\text { STAB A - } \\
\text { Carl }\end{array}$ \\
\hline & Fig $08(8)$ & IGF 104810 & $1262 \mathrm{C} 11-5,131-132$ & IGF 104809-1 & $\begin{array}{l}\text { STAB A - } \\
\text { Carl }\end{array}$ \\
\hline & Fig $08(9)$ & IGF 104811 & $1262 \mathrm{C} 11-5,131-132$ & IGF 104809-1 & $\begin{array}{l}\text { STAB A - } \\
\text { Carl }\end{array}$ \\
\hline & $\begin{array}{l}\text { Fig } 08 \\
(10)\end{array}$ & IGF 104812 & 1209B 25-1, 142-143 & IGF 104803-1 & STAB 12 \\
\hline & Fig 09 (1) & IGF 104813 & 1209B $25-1,142-143$ & IGF 104803-1 & STAB 12 \\
\hline & Fig 09 (2) & IGF 104814 & $1262 \mathrm{C} 11-5,131-132$ & IGF 104809-1 & $\begin{array}{l}\text { STAB A - } \\
\text { Carl }\end{array}$ \\
\hline \multirow[t]{5}{*}{ Gomphiolithus magnicordis } & Fig 09 (3) & IGF 104815 & 1209B 25-2, 20-21 & IGF 104815-1 & Smear slide \\
\hline & Fig $09(4)$ & IGF 104816 & 1209B 25-1, 142-143 & IGF 104803-1 & STAB 12 \\
\hline & Fig $09(5)$ & IGF 104817 & Bottaccione 11.14 & IGF $104817-1$ & Smear slide \\
\hline & Fig $09(6)$ & IGF 104818 & 1209B 25-1, 142-143 & IGF 104803-1 & STAB 12 \\
\hline & Fig $09(7)$ & IGF 104819 & Contessa Highway $10.07 \mathrm{~m}$ & IGF 104819-1 & Smear slide \\
\hline \multirow[t]{6}{*}{ Diantholitha alata } & Fig $11(1)$ & IGF 104820 & 1209C 14-1, 116-117 & IGF 104820-1 & STAB 7 \\
\hline & Fig $11(2)$ & IGF 104821 & $1262 \mathrm{C} 11-2,56-57$ & IGF 104821-1 & $\begin{array}{l}\text { STAB R - } \\
\text { Carl }\end{array}$ \\
\hline & Fig $11(3)$ & IGF 104822 & $1262 \mathrm{C} 11-2,56-57$ & IGF 104821-1 & $\begin{array}{l}\text { STAB R - } \\
\text { Carl }\end{array}$ \\
\hline & Fig $11(4)$ & IGF 104823 & 1262B 20-5, 2-3 & IGF 104823-1 & Smear slide \\
\hline & Fig $11(5)$ & IGF 104824 & 1262B 20-5, 5-6 & IGF 104824-1 & Smear slide \\
\hline & Fig $11(6)$ & IGF 104825 & 1209C 14-1, 84-85 & IGF $104825-1$ & STAB 2 \\
\hline \multirow[t]{11}{*}{ Diantholitha magnolia } & Fig $12(1)$ & IGF 104826 & $1262 \mathrm{C} 11-2,56-57$ & IGF 104826-1 & STAB 3 \\
\hline & Fig $12(2)$ & IGF 104827 & $1262 \mathrm{C} 11-2,56-57$ & IGF 104826-1 & STAB 3 \\
\hline & Fig $12(3)$ & IGF 104828 & 1209C 14-1,116-117 & IGF 104820-1 & STAB 7 \\
\hline & Fig $12(4)$ & IGF 104829 & 1209C 14-1,116-117 & IGF 104820-1 & STAB 7 \\
\hline & Fig $12(5)$ & IGF 104830 & Qreiya +8.3 & IGF 104830-1 & Smear slide \\
\hline & Fig $12(6)$ & IGF 104831 & Qreiya +8.6 & IGF 104831-1 & Smear slide \\
\hline & Fig $12(7)$ & IGF 104832 & Qreiya +8.3 & IGF 104832-1 & Smear slide \\
\hline & Fig $12(8)$ & IGF 104833 & Qreiya +8.3 & IGF 104833-1 & Smear slide \\
\hline & Fig $12(9)$ & IGF 104834 & $1262 \mathrm{C} 11-2,56-57$ & IGF 104834-1 & $\begin{array}{l}\text { STAB C - } \\
\text { Carl }\end{array}$ \\
\hline & $\begin{array}{l}\text { Fig } 12 \\
(10)\end{array}$ & IGF 104835 & $1262 \mathrm{C} 11-2,56-57$ & IGF 104834-1 & $\begin{array}{l}\text { STAB C - } \\
\text { Carl }\end{array}$ \\
\hline & $\begin{array}{l}\text { Fig } 12 \\
(11)\end{array}$ & IGF 104836 & $1262 \mathrm{C} 11-2,56-57$ & IGF 104834-1 & $\begin{array}{l}\text { STAB C - } \\
\text { Carl }\end{array}$ \\
\hline
\end{tabular}




\begin{tabular}{|c|c|c|c|c|c|}
\hline Diantholitha ef D. mariposa & $\begin{array}{l}\text { Fig } 9(8), \\
\text { Fig 12 } \\
(12,14)\end{array}$ & IGF 104837 & $1262 \mathrm{C} 11-2,56-57$ & IGF 104837-1 & $\begin{array}{l}\text { STAB B - } \\
\text { Carl }\end{array}$ \\
\hline & $\begin{array}{l}\text { Fig 12 } \\
(13)\end{array}$ & IGF 104838 & $1262 \mathrm{C} 10-6,23-24$ & IGF 104834-1 & $\begin{array}{l}\text { STAB C - } \\
\text { Carl }\end{array}$ \\
\hline Diantholitha ef $D$. mariposa & $\begin{array}{l}\text { Fig 09 } \\
(9,11,12)\end{array}$ & DST A158-7.1 a & Qreiya+7.1 & DST A158-7.1 & Smear slide \\
\hline \multirow[t]{2}{*}{ Diantholitha mariposa } & $\begin{array}{l}\text { Fig } 09 \\
(11)\end{array}$ & DST A158-7.7 a & Qreiya +7.7 & DST A158-7.7 & Smear slide \\
\hline & \begin{tabular}{|l|} 
Fig 09 \\
$(12)$ \\
\end{tabular} & \begin{tabular}{|l} 
DST A158 -9.42 \\
$\mathrm{a}$
\end{tabular} & Qreiya +9.42 & \begin{tabular}{|l|} 
DST A158 - \\
9.42 \\
\end{tabular} & Smear slide \\
\hline \multirow[t]{8}{*}{ Diantholitha pilula } & Fig $13(1)$ & $\begin{array}{l}\text { IGF 104335- } \\
\text { Holotype }\end{array}$ & 1209C 14-1, 116-117 & IGF 104820-1 & STAB 7 \\
\hline & Fig $13(2)$ & DST $104822 \mathrm{a}$ & $1262 \mathrm{C} 11-2,56-57$ & IGF 104821-1 & \begin{tabular}{|l|} 
STAB R - \\
Carl
\end{tabular} \\
\hline & Fig $13(3)$ & DST $104820 \mathrm{a}$ & 1209C $14-1,116-117$ & IGF 104820-1 & STAB 7 \\
\hline & Fig $13(4)$ & \begin{tabular}{|l|} 
IGF 104719 - \\
Paratype \\
\end{tabular} & $1262 \mathrm{C} 10-6,23-24$ & IGF 104719-1 & \begin{tabular}{|l} 
STAB B - \\
Carl \\
\end{tabular} \\
\hline & Fig $13(5)$ & DST $104721 \mathrm{a}$ & $1209 \mathrm{C} 14-2,50-51$ & IGF 104721-1 & STAB 1 \\
\hline & Fig $13(6)$ & DST $104820 \mathrm{~b}$ & 1209C 14-1, 116-117 & IGF 104820-1 & STAB 7 \\
\hline & Fig $13(7)$ & DST $104721 \mathrm{~b}$ & $1209 \mathrm{C} 14-2,50-51$ & IGF 104721-1 & STAB 1 \\
\hline & Fig $13(8)$ & DST 104820c & 1209C $14-1,116-117$ & IGF 104820-1 & STAB 7 \\
\hline \multirow[t]{5}{*}{ Diantholitha toquea } & Fig $11(7)$ & \begin{tabular}{|l|} 
IGF 104720 - \\
Holotype \\
\end{tabular} & $1262 \mathrm{C} 11-2,56-57$ & IGF 104720-1 & \begin{tabular}{|l|} 
STAB I - \\
Carl
\end{tabular} \\
\hline & Fig $11(8)$ & IGF 104838 & $1262 \mathrm{C} 10-6,23-24$ & IGF 104719-1 & $\begin{array}{l}\text { STAB B - } \\
\text { Carl }\end{array}$ \\
\hline & Fig $11(9)$ & $\begin{array}{l}\text { IGF 104721 - } \\
\text { Paratype }\end{array}$ & $1209 \mathrm{C} 14-2,50-51$ & IGF 104721-1 & STAB 1 \\
\hline & $\begin{array}{l}\text { Fig } 11 \\
(10)\end{array}$ & DST $104721 \mathrm{c}$ & 1209C $14-2,50-51$ & IGF 104721-1 & STAB 1 \\
\hline & $\begin{array}{l}\text { Fig 11 } \\
(11) \\
\end{array}$ & DST $104721 \mathrm{~d}$ & 1209C $14-2,50-51$ & IGF 104721-1 & STAB 1 \\
\hline \multirow[t]{3}{*}{ Lithoptychius chowii } & Fig $15(1)$ & DST $104724 \mathrm{a}$ & 1209C $14-1,56-57$ & IGF 104724-1 & STAB S \\
\hline & Fig $15(2)$ & DST $104729 \mathrm{a}$ & $1209 \mathrm{C} 14-1,56-57$ & IGF 104729-1 & STAB 13 \\
\hline & Fig $15(3)$ & DST $104825 \mathrm{a}$ & $1209 \mathrm{C} 14-1,84-85$ & IGF 104825 & STAB 2 \\
\hline \multirow[t]{3}{*}{ Lithoptychius collaris } & Fig $15(4)$ & DST $104820 \mathrm{~d}$ & 1209C 14-1, 116-117 & IGF 104820-1 & STAB 7 \\
\hline & Fig $15(5)$ & DST $104820 \mathrm{e}$ & 1209C $14-1,116-117$ & IGF 104820-1 & STAB 7 \\
\hline & Fig $15(6)$ & DST $104820 \mathrm{f}$ & 1209C $14-1,116-117$ & IGF 104820-1 & STAB 7 \\
\hline \multirow[t]{3}{*}{ Lithoptychius felis } & Fig $16(5)$ & DST $104729 \mathrm{~b}$ & $1209 \mathrm{C} 14-1,56-57$ & IGF 104729-1 & STAB 13 \\
\hline & Fig $16(6)$ & DST-SM-110 a & $1262 \mathrm{C} 10-6,23-24$ & DST-SM-110 & \begin{tabular}{|l} 
STAB Q - \\
Carl
\end{tabular} \\
\hline & Fig $16(7)$ & \begin{tabular}{|l} 
DST-A171- \\
$12236 \mathrm{a}$ \\
\end{tabular} & 1209C $14-1,116-117$ & $\begin{array}{l}\text { DST-A171- } \\
12236\end{array}$ & Smear slide \\
\hline \multirow[t]{3}{*}{ Lithoptychius galeottii } & Fig $16(1)$ & $\begin{array}{l}\text { IGF } 104722 \text { - } \\
\text { Holotype }\end{array}$ & 1209C $14-1,56-57$ & IGF 104724-1 & STAB S \\
\hline & Fig $16(2)$ & $\begin{array}{l}\text { IGF 104723 - } \\
\text { Paratype }\end{array}$ & 1209B 24-5,8-9 & IGF 104723-1 & STAB10 \\
\hline & Fig $16(3)$ & DST-SM-150 a & 1267B 29-5,84-85 & DST-SM-150 & $\begin{array}{l}\text { STAB 5 - } \\
\text { Carl }\end{array}$ \\
\hline \multirow[t]{3}{*}{ Lithoptychius maioranoae } & Fig $18(1)$ & \begin{tabular}{|l|} 
IGF 104725 - \\
Paratype \\
\end{tabular} & 1209C $14-1,56-57$ & IGF 104724-1 & STAB S \\
\hline & Fig $18(2)$ & $\begin{array}{l}\text { IGF } 104724 \text { - } \\
\text { Holotype }\end{array}$ & $1209 \mathrm{C} 14-1,56-57$ & IGF 104724-1 & STAB S \\
\hline & Fig $18(3)$ & DST $104724 \mathrm{~b}$ & 1209C $14-1,56-57$ & IGF 104724-1 & STAB S \\
\hline \multirow[t]{3}{*}{ Lithoptychius schmitzii } & Fig $16(8)$ & DST $104825 \mathrm{~b}$ & 1209C $14-1,84-85$ & IGF 104825-1 & STAB 2 \\
\hline & Fig $16(9)$ & \begin{tabular}{|l} 
DST-A171- \\
$12243 \mathrm{a}$ \\
\end{tabular} & 1209C $14-1,80-81$ & \begin{tabular}{|l|} 
DST-A171- \\
12243 \\
\end{tabular} & Smear slide \\
\hline & Fig $17(1)$ & DST $104825 \mathrm{c}$ & $1209 \mathrm{C} 14-1,84-85$ & IGF 104825-1 & STAB 2 \\
\hline
\end{tabular}




\begin{tabular}{|c|c|c|c|c|c|}
\hline & Fig 17 (2) & DST $104729 \mathrm{c}$ & $1209 \mathrm{C} 14-1,56-57$ & IGF 104729-1 & STAB 13 \\
\hline & Fig 17 (3) & DST-SM-150 b & 1267B 29-5, 84-85 & DST-SM-150 & \begin{tabular}{|l|} 
STAB 5 - \\
Carl \\
\end{tabular} \\
\hline & Fig 17 (4) & DST $104825 \mathrm{~d}$ & 1209C $14-1,84-85$ & IGF 104825-1 & STAB 2 \\
\hline & Fig $17(5)$ & DST-SM-110 b & $1262 \mathrm{C} 10-6,23-24$ & DST-SM-110 & $\begin{array}{l}\text { STAB Q - } \\
\text { Carl }\end{array}$ \\
\hline & Fig 17 (6) & DST-SM-110 c & $1262 \mathrm{C} 10-6,23-24$ & DST-SM-110 & \begin{tabular}{|l} 
STAB Q - \\
Carl \\
\end{tabular} \\
\hline & Fig 17 (7) & DST-SM- $150 \mathrm{c}$ & 1267B 29-5, 84-85 & DST-SM-150 & \begin{tabular}{|l} 
STAB 5 - \\
Carl
\end{tabular} \\
\hline & Fig $17(8)$ & DST-SM- $150 \mathrm{~d}$ & 1267B 29-5, 84-85 & DST-SM-150 & \begin{tabular}{|l} 
STAB 5 - \\
Carl \\
\end{tabular} \\
\hline & Fig $17(9)$ & DST-SM-110 d & $1262 \mathrm{C} 10-6,23-24$ & DST-SM-110 & \begin{tabular}{|l} 
STAB Q - \\
Carl
\end{tabular} \\
\hline & \begin{tabular}{|l|} 
Fig 17 \\
$(10)$
\end{tabular} & \begin{tabular}{|l} 
DST-B110- \\
$9151 \mathrm{a}$ \\
\end{tabular} & $1262 \mathrm{C} 10-6,23-24$ & \begin{tabular}{|l} 
DST-B110- \\
9151 \\
\end{tabular} & Smear slide \\
\hline & \begin{tabular}{|l} 
Fig 17 \\
$(11)$
\end{tabular} & \begin{tabular}{|l} 
DST-B110- \\
$9151 \mathrm{~b}$
\end{tabular} & $1262 \mathrm{C} 10-6,23-24$ & \begin{tabular}{|l} 
DST-B110- \\
9151
\end{tabular} & Smear slide \\
\hline \multirow[t]{4}{*}{ Lithoptychius ulii } & Fig $18(4)$ & DST $104724 \mathrm{c}$ & $1209 \mathrm{C} 14-1,56-57$ & IGF 104724-1 & STAB S \\
\hline & Fig 18(5) & DST $104721 \mathrm{e}$ & 1267A 29-1, 60-61 & IGF 104721-1 & STAB 1 \\
\hline & Fig $18(6)$ & DST $104729 \mathrm{~d}$ & 1209C $14-1,56-57$ & IGF 104729-1 & STAB 13 \\
\hline & Fig $18(7)$ & DST-SM-170 & $11937-4,135-136$ & DST-SM-170-1 & STAB 17 \\
\hline \multirow{3}{*}{ Lithoptychius varolii } & Fig $19(1)$ & DST $104724 \mathrm{~d}$ & $1209 \mathrm{C} 14-1,56-57$ & IGF 104724-1 & STAB S \\
\hline & Fig $19(2)$ & \begin{tabular}{|l} 
DST-A171- \\
12248 \\
\end{tabular} & 1209C 14-1, 56-57 & \begin{tabular}{|l} 
DST-A171- \\
$12248-1$ \\
\end{tabular} & Smear slide \\
\hline & Fig $19(3)$ & DST-A152-95 & Contessa Road $9.50 \mathrm{~m}$ & $\begin{array}{l}\text { DST-A152-95- } \\
1\end{array}$ & Smear slide \\
\hline \multirow[t]{6}{*}{ Tectulithus janii } & Fig $19(4)$ & DST $104727 \mathrm{a}$ & 356Z 23R-1W, 70-71 & IGF 104727-1 & STAB R \\
\hline & Fig $19(5)$ & DST $104727 \mathrm{~b}$ & 356Z 23R-1W, 70-71 & IGF 104727-1 & STAB R \\
\hline & Fig $19(6)$ & DST $104727 \mathrm{c}$ & 356Z 23R-1W, 70-71 & IGF 104727-1 & STAB R \\
\hline & Fig $19(7)$ & DST-SM-155 a & 356Z 23R-1W, 70-71 & DST-SM-155 & STAB 15 \\
\hline & Fig $19(8)$ & DST $104723 \mathrm{a}$ & 1209B 24-5,8-9 & IGF 104723-1 & STAB 10 \\
\hline & Fig $19(9)$ & DST $104723 \mathrm{~b}$ & 1209B 24-5,8-9 & IGF 104723-1 & STAB 10 \\
\hline \multirow[t]{5}{*}{ Tectulithus merloti } & Fig $21(3)$ & DST-SM-60 a & $1262 \mathrm{C} 10-4,131-132$ & DST-SM-60 & STAB 6 \\
\hline & Fig $21(4)$ & DST-SM-140 a & $1262 \mathrm{C} 10-3,74-75$ & DST-SM-140 & STAB 14 \\
\hline & Fig $21(5)$ & DST-SM-140 b & $1262 \mathrm{C} 10-3,74-75$ & DST-SM-140 & STAB 14 \\
\hline & Fig $21(6)$ & DST-SM-140 c & $1262 \mathrm{C} 10-3,74-75$ & DST-SM-140 & STAB 14 \\
\hline & Fig $21(7)$ & DST-SM-160 a & $11937-4,120-121$ & DST-SM-160 & STAB Z \\
\hline Tectulithus cf. T. merloti & Fig $21(2)$ & DST-SM-160 b & $11937-4,120-121$ & DST-SM-160 & STAB Z \\
\hline \multirow[t]{3}{*}{ Tectulithus pagodiformis } & Fig 22(1) & $\begin{array}{l}\text { IGF } 104726 \text { - } \\
\text { Holotype }\end{array}$ & 356Z 23R-1W, 70-71 & IGF 104727-1 & STAB R \\
\hline & Fig $22(2)$ & $\begin{array}{l}\text { IGF } 104727 \text { - } \\
\text { Paratype }\end{array}$ & 356Z 23R-1W, 70-71 & IGF $104727-1$ & STAB R \\
\hline & Fig $22(3)$ & DST-SM-155 b & 356Z 23R-1W, 70-71 & DST-SM-155 & STAB 15 \\
\hline $\begin{array}{l}\text { Tectulithus cf. } T . \\
\text { pagodiformis }\end{array}$ & Fig $21(1)$ & DST $104727 \mathrm{~d}$ & 356Z 23R-1W, 70-71 & IGF 104727-1 & STAB R \\
\hline \multirow[t]{3}{*}{ Tectulithus pileatus } & Fig $22(4)$ & DST-SM-60 b & $1262 \mathrm{C} 10-4,131-132$ & DST-SM-60 & STAB 6 \\
\hline & Fig $22(5)$ & DST-SM-60 c & $1262 \mathrm{C} 10-4,131-132$ & DST-SM-60 & STAB 6 \\
\hline & Fig $22(6)$ & DST $104723 \mathrm{c}$ & 1209B 24-5,8-9 & IGF 104723-1 & STAB 10 \\
\hline \multirow[t]{2}{*}{ Tectulithus stegastos } & Fig $22(7)$ & DST 104724 e & $1209 \mathrm{C} 14-1,56-57$ & IGF 104724-1 & STAB S \\
\hline & Fig $22(8)$ & DST-SM-158 a & 1209B 24-5,8-9 & DST-SM-158 & STAB V \\
\hline \multirow[t]{2}{*}{ Tectulithus stonehengei } & Fig $23(1)$ & DST-SM-60 d & $1262 \mathrm{C} 10-4,131-132$ & DST-SM-60 & STAB 6 \\
\hline & Fig $23(2)$ & DST-SM-140 d & $1262 \mathrm{C} 10-3,74-75$ & DST-SM-140 & STAB 14 \\
\hline
\end{tabular}




\begin{tabular}{|c|c|c|c|c|c|}
\hline & Fig 23 (3) & DST $104723 \mathrm{~d}$ & 1209B 24-5,8-9 & IGF 104723 & STAB 10 \\
\hline \multirow[t]{4}{*}{ Fasciculithus billii } & Fig 23 (4) & DST-SM-60 e & $1262 \mathrm{C} 10-4,131-132$ & DST-SM-60 & STAB 6 \\
\hline & Fig $23(5)$ & DST $104723 \mathrm{e}$ & 1209B 24-5,8-9 & IGF 104723 & STAB 10 \\
\hline & Fig $23(6)$ & DST-SM-140 e & $1262 \mathrm{C} 10-3,74-75$ & DST-SM-140 & STAB 14 \\
\hline & Fig $23(7)$ & DST-SM-159 a & $1262 \mathrm{C} 10-4,131-132$ & DST-SM-159 & STAB W \\
\hline \multirow[t]{4}{*}{ Fasciculithus sp. A } & Fig $24(1)$ & DST-SM-140 f & $1262 \mathrm{C} 10-3,74-75$ & DST-SM-140 & STAB 14 \\
\hline & Fig $24(2)$ & DST-SM-140 g & $1262 \mathrm{C} 10-3,74-75$ & DST-SM-140 & STAB 14 \\
\hline & Fig 24 (3) & DST-SM-140 h & $1262 \mathrm{C} 10-3,74-75$ & DST-SM-140 & STAB 14 \\
\hline & Fig $24(4)$ & DST-SM-140 i & $1262 \mathrm{C} 10-3,74-75$ & DST-SM-140 & STAB 14 \\
\hline \multirow[t]{3}{*}{ Fasciculithus realeae } & Fig $24(5)$ & $\begin{array}{l}\text { IGF 104728 - } \\
\text { Holotype }\end{array}$ & $1209 \mathrm{C} 14-1,56-57$ & IGF 104729-1 & STAB 13 \\
\hline & Fig $24(6)$ & $\begin{array}{l}\text { IGF } 104729 \text { - } \\
\text { Paratype }\end{array}$ & $1209 \mathrm{C} 14-1,56-57$ & IGF 104724-1 & STAB S \\
\hline & Fig $24(7)$ & DST $104724 \mathrm{f}$ & 1209C $14-1,56-57$ & IGF 104724-1 & STAB S \\
\hline \multirow[t]{5}{*}{ Fasciculithus vertebratoides } & Fig $26(1)$ & DST 104727 e & 356Z 23R-1W, 70-71 & IGF 104727-1 & STAB R \\
\hline & Fig $26(2)$ & DST $104727 \mathrm{f}$ & 356Z 23R-1W, 70-71 & IGF 104727-1 & STAB R \\
\hline & Fig 26 (3) & DST-SM-110 e & $1262 \mathrm{C} 10-6,23-24$ & DST-SM-110 & $\begin{array}{l}\text { STAB Q - } \\
\text { Carl }\end{array}$ \\
\hline & Fig $26(4)$ & DST-SM-165 a & $11937-4,40-41$ & DST-SM-165 & STAB T \\
\hline & Fig $26(5)$ & DST-SM-155 c & 356Z 23R-1W, 70-71 & DST-SM-155 & STAB 15 \\
\hline
\end{tabular}

Table S2. Calcareous nannofossil zonation and reference for the biostratigraphic determination of all the samples investigated in this study.

\begin{tabular}{|l|c|c|c|l|}
\hline \multirow{2}{*}{ Site/Section } & \multirow{2}{*}{ DEPTH } & \multicolumn{2}{|c|}{$\begin{array}{c}\text { Calcareous Nannofossil } \\
\text { Zonation }\end{array}$} & \multicolumn{1}{|c|}{ References } \\
\cline { 3 - 4 } & & Martini, 1971 & $\begin{array}{c}\text { Okada \& } \\
\text { Bukry, 1980 }\end{array}$ & \\
\hline 1262C 11-5, 131-132 & $201.34(\mathrm{mcd})$ & NP4 & CP3 & Monechi et al., 2013 \\
\hline 1262C 11-2, 56-57 & $196.09(\mathrm{mcd})$ & NP4 & CP3 & Monechi et al., 2013 \\
\hline 1262B 20-5, 5-6 & $195.29(\mathrm{mcd})$ & NP4 & CP3 & Monechi et al., 2013 \\
\hline 1262B 20-5, 2-3 & $195.26(\mathrm{mcd})$ & NP4 & CP3 & Monechi et al., 2013 \\
\hline 1262C 10-6, 23-24 & $190.66(\mathrm{mcd})$ & NP4 & CP3 & Monechi et al., 2013 \\
\hline 1262C 10-4,131-132 & $188.74(\mathrm{mcd})$ & NP5 & CP4 & Monechi et al., 2013 \\
\hline 1262C 10-3,74-75 & $186.67(\mathrm{mcd})$ & NP5 & CP4 & Monechi et al., 2013 \\
\hline 1267A 29-1, 60-61 & $297(\mathrm{mcd})$ & NP2-NP4 & & Zachos et al., 2004 \\
\hline 1267B 29-5, 84-85 & $295.04(\mathrm{mcd})$ & NP2-NP4 & & Zachos et al., 2004 \\
\hline 1209B 25-1, 142-143 & $251.25(\mathrm{rmcd})$ & NP4 & CP3 & Bralower, 2005 \\
\hline 1209B 25-2, 20-21 & $251.53(\mathrm{rmcd})$ & NP4 & CP3 & Bralower, 2005 \\
\hline 1209C 14-2, 50-51 & $249.325(\mathrm{rmcd})$ & NP4 & CP3 & Bralower, 2005 \\
\hline 1209C 14-1, 116-117 & $248.384(\mathrm{rmcd})$ & NP4 & CP3 & Bralower, 2005 \\
\hline 1209C 14-1, 84-85 & $248.04(\mathrm{rmcd})$ & NP4 & CP3 & Bralower, 2005 \\
\hline 1209C 14-1, 80-81 & $248(\mathrm{rmcd})$ & NP4 & CP3 & Bralower, 2005 \\
\hline 1209C 14-1, 56-57 & $247.76(\mathrm{rmcd})$ & NP4 & CP3 & Bralower, 2005 \\
\hline 1209B 24-5,8-9 & $244.81(\mathrm{rmcd})$ & NP4 & CP3 & Bralower, 2005 \\
\hline 356Z 23R-1W, 70-71 & $352.2(\mathrm{mbsf})$ & NP5 & CP4 & Perch-Nielsen, 1977 \\
\hline 119 37-4, 135-136 & $667.85(\mathrm{mbsf})$ & NP5 & CP4 & Perch-Nielsen, 1971 \\
\hline 119 37-4, 120-121 & $667.7(\mathrm{mbsf})$ & NP5 & CP4 & Perch-Nielsen, 1971 \\
\hline
\end{tabular}




\begin{tabular}{|c|c|c|c|c|}
\hline $11937-4,40-41$ & 666.9 (mbsf) & NP5 & $\mathrm{CP} 4$ & Perch-Nielsen, 1971 \\
\hline Bottaccione 10.60 & $10.60 \mathrm{~m}$ above $\mathrm{K} / \mathrm{Pg}$ & NP4 & $\mathrm{CP} 3$ & Galeotti et al., 2015 \\
\hline Bottaccione 11.14 & $\begin{array}{c}11.14 \mathrm{~m} \text { above } \\
\mathrm{K} / \mathrm{Pg}\end{array}$ & NP4 & $\mathrm{CP} 3$ & Galeotti et al., 2015 \\
\hline Bottaccione 11.38 & $11.38 \mathrm{~m}$ above $\mathrm{K} / \mathrm{Pg}$ & NP4 & $\mathrm{CP} 3$ & Galeotti et al., 2015 \\
\hline $\begin{array}{l}\text { Contessa Highway } \\
10.63\end{array}$ & $\begin{array}{c}10.63 \mathrm{~m} \text { above } \\
\mathrm{K} / \mathrm{Pg}\end{array}$ & NP4 & $\mathrm{CP} 3$ & Galeotti et al., 2015 \\
\hline $\begin{array}{l}\text { Contessa Highway } \\
11.35\end{array}$ & $11.35 \mathrm{~m}$ above $\mathrm{K} / \mathrm{Pg}$ & NP4 & $\mathrm{CP} 3$ & Galeotti et al., 2015 \\
\hline Contessa Road 9,50 & $9.50 \mathrm{~m}$ above $\mathrm{K} / \mathrm{Pg}$ & NP4 & $\mathrm{CP} 3$ & Galeotti et al., 2015 \\
\hline Qreiya +7.1 & meter 7.1 & NP4 & $\mathrm{CP} 3$ & $\begin{array}{l}\text { Monechi et al., 2013; Aubry et al., } \\
2012\end{array}$ \\
\hline Qreiya +7.7 & meter 7.7 & NP4 & $\mathrm{CP} 3$ & $\begin{array}{l}\text { Monechi et al., 2013; Aubry et al., } \\
2012\end{array}$ \\
\hline Qreiya +8.3 & meter 8.3 & NP4 & $\mathrm{CP} 3$ & $\begin{array}{l}\text { Monechi et al., 2013; Aubry et al., } \\
2012\end{array}$ \\
\hline Qreiya +8.6 & meter 8.6 & NP4 & $\mathrm{CP} 3$ & $\begin{array}{l}\text { Monechi et al., 2013; Aubry et al., } \\
2012\end{array}$ \\
\hline Qreiya +9.42 & meter 9.4 & NP4 & $\mathrm{CP} 3$ & $\begin{array}{l}\text { Monechi et al., 2013; Aubry et al., } \\
2012\end{array}$ \\
\hline
\end{tabular}

Table S3. Morphometric measurements of Gomphiolithus acquired for this study.

\begin{tabular}{|c|c|c|c|c|}
\hline \multirow[b]{2}{*}{ Gomphiolithus } & \multirow[b]{2}{*}{ Specimen } & \multirow[b]{2}{*}{ Sample } & \multicolumn{2}{|c|}{ Column } \\
\hline & & & height $(\mu \mathrm{m})$ & width $(\mu \mathrm{m})$ \\
\hline \multirow{11}{*}{ G. magnus } & Fig $8(1)$ & 1209B 25-1, 142-143 & $7(7.1)$ & $8(8.2)$ \\
\hline & Fig $8(2)$ & 1209B 25-1, 142-143 & 5.2 & 6.5 \\
\hline & Fig 8 (3) & 1209B 25-1, 142-143 & 8.1 & 9.9 \\
\hline & Fig 8 (4) & Contessa Highway $11,35 \mathrm{~m}$ & 11.3 & 12.9 \\
\hline & Fig 8 (5) & Bottaccione $10,60 \mathrm{~m}$ & 10.7 & 11.8 \\
\hline & Fig 8 (6) & Bottaccione $11,38 \mathrm{~m}$ & 9.2 & 9.7 \\
\hline & Fig 8 (7) & 1262 C $11-5,131-132$ & 7.6 & 9.5 \\
\hline & Fig $8(8)$ & $1262 \mathrm{C} 11-5,131-132$ & 7.4 & 8.2 \\
\hline & Fig 8 (9) & 1262 C 11-5, 131-132 & 5.5 & 7.4 \\
\hline & Fig $8(10)$ & 1209B 25-1, 142-143 & 8.6 & 10.7 \\
\hline & Fig 8 (11) & 1262 C 11-5, 131-132 & 9.3 & 11.0 \\
\hline \multirow[t]{5}{*}{ G. magnicordis } & Fig 9 (3) & 1209B 25-2, 20-21 & 7.8 & 10.1 \\
\hline & Fig 9 (4) & 1209B 25-1, 142-143 & 7.9 & 9.6 \\
\hline & Fig 9(5) & Bottaccione $11,14 \mathrm{~m}$ & 7.0 & 8.1 \\
\hline & Fig 9 (6) & 1209B 25-1, 142-143 & 6.5 & 8.7 \\
\hline & Fig 9 (7) & Contessa Highway $10,63 \mathrm{~m}$ & 8.0 & 9.2 \\
\hline
\end{tabular}

Table S4. Morphometric measurements of Diantholitha acquired for this study.

\begin{tabular}{|c|c|c|c|c|c|c|c|}
\hline \multirow[b]{2}{*}{ Diantholitha } & \multirow[b]{2}{*}{ Specimen } & \multirow[b]{2}{*}{ Sample } & \multicolumn{2}{|c|}{ Column } & \multicolumn{2}{|c|}{ Biretta } & \multirow{2}{*}{$\begin{array}{c}\text { Total } \\
\text { height } \\
(\mu \mathrm{m})\end{array}$} \\
\hline & & & $\begin{array}{c}\text { height } \\
(\mu \mathrm{m})\end{array}$ & $\begin{array}{c}\text { width } \\
(\mu \mathrm{m})\end{array}$ & $\begin{array}{c}\text { height } \\
(\mu \mathrm{m})\end{array}$ & $\begin{array}{c}\text { width } \\
(\mu \mathrm{m})\end{array}$ & \\
\hline D. alata & Fig $11(1)$ & 1209C 14-1, 116-117 & 1.9 & 6.4 & 3.7 & 6.3 & 5.6 \\
\hline
\end{tabular}




\begin{tabular}{|c|c|c|c|c|c|c|c|}
\hline & Fig $11(2)$ & $1209 \mathrm{C} 14-1,84-85$ & 1.5 & 4.2 & 4.3 & 2.2 & 5.8 \\
\hline & Fig $11(4)$ & 1262B 20-5, 2-3 & 2.2 & 6.8 & 4.3 & 7.0 & 6.5 \\
\hline & Fig $11(5)$ & 1262B 20-5, 5-6 & 2.6 & 8.5 & 5.1 & 8.2 & 7.7 \\
\hline \multirow[t]{9}{*}{ D. magnolia } & Fig $12(1)$ & $1262 \mathrm{C} 11-2,56-57$ & 2.2 & 6.1 & 3.7 & 6.6 & 5.9 \\
\hline & Fig $12(2)$ & $1262 \mathrm{C} 11-2,56-57$ & 2.0 & 5.8 & 3.5 & 6.4 & 5.4 \\
\hline & Fig $12(3)$ & 1209C 14-1, 116-117 & 2.2 & 6.5 & 4.6 & 7.0 & 6.8 \\
\hline & Fig $12(4)$ & 1209C 14-1,116-117 & 1.3 & 4.7 & 3.0 & 5.2 & 4.4 \\
\hline & Fig $12(5)$ & Qreiya $+8,3$ & 2.6 & 6.4 & 4.2 & 6.7 & 6.8 \\
\hline & Fig $12(6)$ & Qreiya $+8,6$ & 2.3 & 6.1 & 3.4 & 5.8 & 5.7 \\
\hline & Fig $12(7)$ & Qreiya $+8,3$ & 2.4 & 5.5 & 3.7 & 6.3 & 6.1 \\
\hline & Fig $12(8)$ & Qreiya $+8,3$ & 2.3 & 5.4 & 3.3 & 5.3 & 5.6 \\
\hline & Fig $12(9)$ & $1262 \mathrm{C} 11-2,56-57$ & 1.7 & 7.6 & 3.0 & 6.2 & 4.7 \\
\hline \multirow{3}{*}{$\begin{array}{l}\text { Diantholiotha } \mathrm{cf} \\
\text { D. mariposa }\end{array}$} & Fig $9(8)$ & 1209C $14-1,116-117$ & 3.1 & 6.6 & 3.2 & 7.0 & 6.3 \\
\hline & Fig 9 (9) & Qreiya $+7,1 \mathrm{~m}$ & 2.6 & 6.3 & 4.5 & 8.5 & 7.1 \\
\hline & Fig $9(10)$ & Qreiya+7,1m & 3.2 & 7.7 & 3.2 & 7.9 & 6.4 \\
\hline \multirow[t]{2}{*}{ D. mariposa } & Fig 9 (11) & Qreiya $+7,7 \mathrm{~m}$ & 3.3 & 6.9 & 4.1 & 7.7 & 7.4 \\
\hline & Fig 9 (12) & Qreiya $+9,42 m$ & 3.0 & 6.8 & 3.8 & 6.6 & 6.8 \\
\hline \multirow[t]{7}{*}{ D. pilula } & Fig 13 (1) & 1209C 14-1, 116-117 & 2.0 & 6.0 & 4.0 & 7.0 & 6.0 \\
\hline & Fig $13(2)$ & $1262 \mathrm{C} 11-2,56-57$ & 2.3 & 6.1 & 3.9 & 7.1 & 6.2 \\
\hline & Fig $13(3)$ & 1209C 14-1, 116-117 & 2.1 & 6.0 & 4.3 & 7.6 & 6.4 \\
\hline & Fig $13(4)$ & $1262 \mathrm{C} 11-2,56-57$ & 1.9 & 6.3 & 3.9 & 6.7 & 5.9 \\
\hline & Fig $13(5)$ & 1209C 14-2, 50-51 & 1.8 & 9.0 & 3.8 & 6.9 & 5.6 \\
\hline & Fig 13 (6) & 1209 14-1, 116-117 & 2.3 & 6.2 & 5.0 & 7.0 & 7.3 \\
\hline & Fig 13 (7) & $1209 \mathrm{C} 14-2,50-51$ & 1.6 & 4.8 & 3.4 & 5.4 & 5.1 \\
\hline \multirow[t]{4}{*}{ D. toquea } & Fig $11(7)$ & $126211-2,56-57$ & 1.9 & 4.8 & 3.8 & 6.1 & 5.7 \\
\hline & Fig $11(9)$ & $1209 \mathrm{C} 14-2,50-51$ & 2.0 & 5.4 & 4.1 & 7.3 & 6.1 \\
\hline & $\begin{array}{l}\text { Fig 11 } \\
(10)\end{array}$ & $120914-2,50-51$ & 2.3 & 6.5 & 4.6 & 7.9 & 6.9 \\
\hline & $\begin{array}{l}\text { Fig } 11 \\
(11)\end{array}$ & $120914-2,50-51$ & 2.4 & 6.9 & 4.9 & 8.8 & 7.3 \\
\hline
\end{tabular}

Table S5. Morphometric measurements of Lithoptychius acquired for this study.

\begin{tabular}{|c|c|c|c|c|c|c|c|c|c|c|}
\hline \multirow[t]{2}{*}{ Lithoptychius } & \multirow[t]{2}{*}{ Specimen } & \multirow[t]{2}{*}{ Sample } & \multicolumn{3}{|c|}{ Column } & \multicolumn{2}{|c|}{ Collaret } & \multicolumn{2}{|c|}{ Calyptra } & \multirow{2}{*}{$\begin{array}{l}\text { Total } \\
\text { height } \\
(\mu \mathrm{m})\end{array}$} \\
\hline & & & $\begin{array}{c}\text { height } \\
(\mu \mathrm{m})\end{array}$ & $\begin{array}{l}\text { prossimal } \\
\text { width } \\
(\mu \mathrm{m})\end{array}$ & $\begin{array}{l}\text { distal } \\
\text { width } \\
(\mu \mathrm{m})\end{array}$ & $\begin{array}{l}\text { height } \\
(\mu \mathrm{m})\end{array}$ & $\begin{array}{c}\text { width } \\
(\mu \mathrm{m})\end{array}$ & $\begin{array}{c}\text { height } \\
(\mu \mathrm{m})\end{array}$ & $\begin{array}{c}\text { width } \\
(\mu \mathrm{m})\end{array}$ & \\
\hline \multirow[t]{3}{*}{ L. chowii } & Fig $15(1)$ & $\begin{array}{l}\text { 1209C 14-1, } \\
56-57\end{array}$ & 2.5 & 3.8 & 4.3 & 0.7 & 3.8 & 1.1 & 4.3 & 4.3 \\
\hline & Fig $15(2)$ & $\begin{array}{l}1209 \mathrm{C} 14-1, \\
56-57\end{array}$ & 2.6 & 4.8 & 6.4 & 0.9 & 5.6 & 1.7 & 4.3 & 5.2 \\
\hline & Fig 15 (3) & $\begin{array}{l}1209 \mathrm{C} 14-1, \\
84-85\end{array}$ & 4.2 & 5.3 & 6.6 & 0.8 & 5.8 & 1.5 & 5.1 & 6.5 \\
\hline \multirow[t]{3}{*}{ L. collaris } & Fig 15 (4) & $\begin{array}{l}\text { 1209C 14-1, } \\
116-117\end{array}$ & 2.0 & 4.6 & 5.0 & 1.5 & 7.3 & 2.2 & 4.5 & 5.7 \\
\hline & Fig $15(5)$ & $\begin{array}{l}\text { 1209C 14-1, } \\
116-117\end{array}$ & 2.2 & 4.7 & 4.9 & 1.3 & 6.1 & 1.8 & 4.1 & 5.3 \\
\hline & Fig 15 (6) & $\begin{array}{l}\text { 1209C 14-1, } \\
116-117\end{array}$ & 2.1 & 3.9 & 4.3 & 1.0 & 5.7 & 1.8 & 3.4 & 4.9 \\
\hline
\end{tabular}




\begin{tabular}{|c|c|c|c|c|c|c|c|c|c|c|}
\hline \multirow[t]{3}{*}{ L.felis } & Fig 16(5) & $\begin{array}{l}1209 \text { C 14-1, } \\
56-57\end{array}$ & 2.6 & 5.5 & 5.5 & 1.0 & 6.1 & 1.8 & 4.7 & 5.3 \\
\hline & Fig $16(6)$ & $\begin{array}{l}1262 \text { C } 10-6, \\
23-24\end{array}$ & 2.8 & 5.1 & 5.6 & 0.7 & 6.2 & 1.7 & 4.0 & 5.2 \\
\hline & Fig $16(7)$ & $\begin{array}{l}1209 \mathrm{C} 14- \\
1,116-117 \\
\end{array}$ & 2.4 & 4.9 & 5.0 & 1.1 & 6.3 & 2.0 & 3.9 & 5.5 \\
\hline \multirow[t]{4}{*}{ L. galeottii } & Fig $16(1)$ & $\begin{array}{l}1209 \text { C 14-1, } \\
56-57\end{array}$ & 3.1 & 5.0 & 6.0 & 0.5 & 6.5 & 2.5 & 4.3 & 6.1 \\
\hline & Fig $16(2)$ & $\begin{array}{l}1209 \mathrm{~B}-24- \\
5,8-9\end{array}$ & 4.7 & 5.8 & 7.0 & 0.7 & 7.2 & 1.5 & 5.2 & 7.0 \\
\hline & Fig $16(3)$ & $\begin{array}{l}1267 \mathrm{~B} 29- \\
5,84-85\end{array}$ & 3.0 & 5.0 & 6.0 & 0.9 & 6.1 & 1.7 & 3.0 & 5.6 \\
\hline & Fig $16(4)$ & $\begin{array}{l}1267 \mathrm{~B} 29- \\
5,84-85\end{array}$ & 3.6 & 5.1 & 6.2 & 0.8 & 6.5 & 2.2 & 4.8 & 6.6 \\
\hline \multirow[t]{3}{*}{ L. maioranoae } & Fig 18 (1) & $\begin{array}{l}1209 \text { C 14-1, } \\
56-57\end{array}$ & 3.0 & 6.0 & 6.8 & 0.5 & 7.0 & 1.5 & 4.3 & 5.5 \\
\hline & Fig $18(2)$ & $\begin{array}{l}1209 \text { C 14-1, } \\
56-57\end{array}$ & 2.6 & 5.6 & 6.1 & 0.9 & 6.6 & 2.1 & 4.6 & 5.6 \\
\hline & Fig 18 (3) & $\begin{array}{l}1209 \mathrm{C}-14- \\
1,56-57 \\
\end{array}$ & 3.1 & 5.6 & 6.8 & 0.9 & 7.6 & 2.1 & 4.9 & 6.0 \\
\hline \multirow[t]{8}{*}{ L. schmitzii } & Fig $16(8)$ & $\begin{array}{l}\text { 1209C 14-1, } \\
84-85\end{array}$ & 2.1 & 4.4 & 4.8 & 1.1 & 6.1 & 1.6 & 3.7 & 4.8 \\
\hline & Fig $16(9)$ & $\begin{array}{l}1209 \text { C 14-1, } \\
80-81\end{array}$ & 2.1 & 5.3 & 5.4 & 1.4 & 6.6 & 1.9 & 4.2 & 5.4 \\
\hline & Fig 17 (1) & $\begin{array}{l}\text { 1209C 14-1, } \\
84-85\end{array}$ & 2.4 & 4.9 & 5.5 & 1.7 & 6.2 & 1.6 & 3.7 & 5.8 \\
\hline & Fig 17 (2) & $\begin{array}{l}1209 \text { C 14-1, } \\
56-57\end{array}$ & 3.1 & 4.3 & 5.1 & 1.2 & 5.5 & 2.0 & 3.5 & 6.2 \\
\hline & Fig $17(5)$ & $\begin{array}{l}1262 \mathrm{C} 10-6, \\
23-24\end{array}$ & 1.6 & 4.2 & 4.6 & 0.9 & 5.3 & 1.3 & 3.1 & 3.8 \\
\hline & Fig 17 (6) & $\begin{array}{l}1262 \mathrm{C} 10-6, \\
23-24\end{array}$ & 1.2 & 3.9 & 4.3 & 0.9 & 4.9 & 1.4 & 3.4 & 3.5 \\
\hline & $\begin{array}{l}\text { Fig } 17 \\
(10)\end{array}$ & $\begin{array}{l}1262 \text { C } 10-6, \\
23-24\end{array}$ & 4.4 & 6.3 & 7.1 & 2.0 & 8.1 & 1.6 & 4.4 & 8.0 \\
\hline & $\begin{array}{l}\text { Fig } 17 \\
(11)\end{array}$ & $\begin{array}{l}1262 \mathrm{C} 10-6, \\
23-24\end{array}$ & 2.6 & 5.8 & 6.2 & 1.1 & 7.0 & 1.1 & 3.0 & 4.7 \\
\hline \multirow[t]{4}{*}{ L. ulii } & Fig $18(4)$ & $\begin{array}{l}1209 \text { C 14-1, } \\
56-57\end{array}$ & 4.3 & 5.9 & 6.2 & 0.8 & 5.9 & 1.2 & 4.5 & 6.2 \\
\hline & Fig $18(5)$ & $\begin{array}{l}\text { 1267A 29-1, } \\
60-61\end{array}$ & 5.5 & 7.6 & 8.0 & 0.8 & 6.8 & 1.5 & 5.7 & 7.7 \\
\hline & Fig $18(6)$ & $\begin{array}{l}1209 \text { C 14-1, } \\
56-57\end{array}$ & 4.7 & 5.7 & 6.9 & 0.7 & 5.7 & 1.4 & 4.8 & 6.7 \\
\hline & Fig $18(7)$ & $\begin{array}{l}11937-4, \\
135-136\end{array}$ & 4.9 & 5.5 & 6.3 & 0.8 & 5.7 & 1.0 & 3.6 & 6.6 \\
\hline \multirow[t]{3}{*}{ L. varolii } & Fig $19(1)$ & $\begin{array}{l}\text { 1209C 14-1, } \\
56-57\end{array}$ & 2.3 & 4.9 & 5.5 & 0.8 & 4.6 & 1.2 & 4.4 & 4.3 \\
\hline & Fig 19 (2) & $\begin{array}{l}1209 \text { C } 14- \\
1,56-57\end{array}$ & 1.8 & 3.7 & 5.4 & 0.7 & 5.6 & 1.7 & 5.4 & 4.1 \\
\hline & Fig 19 (3) & $\begin{array}{l}\text { Contessa } \\
\text { Road } 9,50 \\
\mathrm{~m} \\
\end{array}$ & 2.5 & 4.8 & 5.0 & 1.0 & 5.9 & 2.3 & 4.9 & 5.9 \\
\hline
\end{tabular}

Table S6. Morphometric measurements of Tectulithus acquired for this study.

\begin{tabular}{|c|c|c|c|c|c|c|c|c|}
\hline \multirow[b]{2}{*}{ Tectulithus } & \multirow[b]{2}{*}{ Specimen } & \multirow[b]{2}{*}{ Sample } & \multicolumn{3}{|c|}{ Column } & \multicolumn{2}{|c|}{ Tectum } & \multirow{2}{*}{$\begin{array}{c}\text { Total } \\
\text { height } \\
(\mu \mathrm{m})\end{array}$} \\
\hline & & & $\begin{array}{c}\text { height } \\
(\mu \mathrm{m})\end{array}$ & $\begin{array}{c}\text { prossimal } \\
\text { width } \\
(\mu \mathrm{m})\end{array}$ & $\begin{array}{c}\text { distal } \\
\text { width } \\
(\mu \mathrm{m})\end{array}$ & $\begin{array}{c}\text { height } \\
(\mu \mathrm{m})\end{array}$ & $\begin{array}{c}\text { width } \\
(\mu \mathrm{m})\end{array}$ & \\
\hline T. janii & Fig 19 (4) & 356Z 23R-1W, 70-71 & 4.0 & 3.4 & 5.3 & 2.9 & 7.4 & 7.0 \\
\hline & Fig $19(5)$ & $356 \mathrm{Z} 23 \mathrm{R}-1 \mathrm{~W}, 70-71$ & 4.5 & 3.4 & 5.4 & 3.2 & 7.4 & 7.7 \\
\hline
\end{tabular}




\begin{tabular}{|c|c|c|c|c|c|c|c|c|}
\hline & Fig 19 (6) & 356Z 23R-1W, 70-71 & 3.6 & 3.0 & 5.0 & 2.1 & 6.7 & 5.7 \\
\hline & Fig 19 (7) & $356 \mathrm{Z}$ 23R-1W, 70-71 & 4.0 & 3.6 & 5.2 & 2.4 & 7.4 & 6.5 \\
\hline & Fig $19(8)$ & 1209B 24-5,8-9 & 4.4 & 3.3 & 4.6 & 2.7 & 7.8 & 7.1 \\
\hline & Fig $19(9)$ & 1209B 24-5,8-9 & 3.6 & 2.6 & 4.3 & 2.6 & 6.9 & 6.2 \\
\hline $\begin{array}{l}\text { Tectulithus cf. } T \text {. } \\
\text { pagodiformis }\end{array}$ & Fig $21(1)$ & 356Z 23R-1, 70-7 & 4.5 & 4.9 & 6.5 & 1.6 & 6.0 & 6.1 \\
\hline $\begin{array}{l}\text { Tectulithus cf. } T \text {. } \\
\text { merloti }\end{array}$ & Fig $21(2)$ & $11937-4,120-121$ & 3.2 & 4.0 & 5.0 & 3.3 & 7.2 & 6.5 \\
\hline \multirow[t]{5}{*}{ T. merloti } & Fig $21(3)$ & $1262 \mathrm{C} 10-4,131-132$ & 4.0 & 3.3 & 5.0 & 2.3 & 6.3 & 6.2 \\
\hline & Fig $21(4)$ & $1262 \mathrm{C} \quad 10-3,74-75$ & 2.9 & 3.8 & 5.0 & 2.2 & 6.5 & 5.0 \\
\hline & Fig $21(5)$ & $1262 \mathrm{C} \quad 10-3,74-75$ & 3.3 & 4.2 & 5.1 & 1.9 & 6.3 & 5.2 \\
\hline & Fig 21 (6) & $1262 \mathrm{C} 10-3,74-75$ & 3.5 & 3.6 & 5.5 & 2.1 & 6.7 & 5.6 \\
\hline & Fig $21(7)$ & $11937-4,120-121$ & 2.7 & 2.7 & 4.2 & 2.1 & 6.5 & 4.8 \\
\hline \multirow[t]{3}{*}{ T. pagodiformis } & Fig $22(1)$ & $356 \mathrm{Z} 23 \mathrm{R}-1,70-71$ & 5.4 & 6.4 & 7.2 & 2.0 & 6.5 & 7.4 \\
\hline & Fig $22(2)$ & $356 \mathrm{Z} 23 \mathrm{R}-1,70-72$ & 4.7 & 4.9 & 6.1 & 2.5 & 5.7 & 7.2 \\
\hline & Fig $22(3)$ & $356 \mathrm{Z} 23 \mathrm{R}-1,70-73$ & 5.2 & 6.1 & 7.1 & 2.6 & 3.9 & 7.8 \\
\hline \multirow[t]{3}{*}{ T. pileatus } & Fig $22(4)$ & $356 \mathrm{Z} 23 \mathrm{R}-1,70-74$ & 4.5 & 5.3 & 7.0 & 2.4 & 6.7 & 7.0 \\
\hline & Fig $22(5)$ & $1262 \mathrm{C} 10-4,131-132$ & 4.23 & 4.71 & 6.15 & 2.37 & 5.73 & 6.6 \\
\hline & Fig $22(6)$ & 1209B 24-5, 8-9 & 4.3 & 5.1 & 6.67 & 2.55 & 6.2 & 6.85 \\
\hline \multirow[t]{2}{*}{ T. stegastos } & Fig $22(7)$ & $1209 \mathrm{C} 14-1,56-57$ & 3.25 & 5 & 5.68 & 0.56 & 5.63 & 3.81 \\
\hline & Fig $22(8)$ & 1209B 24-5, 8-9 & 3.3 & 4.72 & 5.86 & 0.9 & 6.15 & 4.2 \\
\hline \multirow[t]{3}{*}{ T. stonehengei } & Fig $23(1)$ & $1262 \mathrm{C} 10-4,131-132$ & 3.5 & 3.23 & 4.66 & 1.61 & 5.58 & 5.11 \\
\hline & Fig 23 (2) & $1262 \mathrm{C} 10-3,74-75$ & 4.14 & 4.53 & 5.58 & 2.25 & 6.36 & 6.39 \\
\hline & Fig 23 (3) & 1209B 24-5,8-9 & 3.05 & 3.51 & 4.45 & 1.79 & 5.84 & 4.84 \\
\hline
\end{tabular}

Table S7. Morphometric measurements of Fasciculithus acquired for this study.

\begin{tabular}{|c|c|c|c|c|c|c|c|c|}
\hline \multirow[b]{2}{*}{ Fasciculithus } & \multirow[b]{2}{*}{ Specimen } & \multirow[b]{2}{*}{ Sample } & \multicolumn{3}{|c|}{ Column } & \multicolumn{2}{|c|}{ Calyptra } & \multirow{2}{*}{$\begin{array}{c}\text { Total } \\
\text { heigh } \\
(\mu \mathrm{m})\end{array}$} \\
\hline & & & $\begin{array}{c}\begin{array}{c}\text { height } \\
(\mu \mathrm{m})\end{array} \\
\end{array}$ & $\begin{array}{c}\text { prossimal } \\
\text { width }(\mu \mathrm{m})\end{array}$ & $\begin{array}{c}\text { distal } \\
\text { width }(\mu \mathrm{m}) \\
\end{array}$ & $\begin{array}{c}\begin{array}{c}\text { height } \\
(\mu \mathrm{m})\end{array} \\
\end{array}$ & $\begin{array}{c}\text { width } \\
(\mu \mathrm{m})\end{array}$ & \\
\hline \multirow[t]{4}{*}{ F. billii } & Fig $23(4)$ & $1262 \mathrm{C} 10-4,131-132$ & 5.5 & 5.5 & 6.8 & 1.5 & 3.2 & 5.5 \\
\hline & Fig $23(5)$ & 1209B 24-5, 8-9 & 6.0 & 6.2 & 6.9 & 1.1 & 3.1 & 7.0 \\
\hline & Fig $23(6)$ & $1262 \mathrm{C} 10-3,74-75$ & 4.2 & 5.9 & 6.8 & 1.1 & 3.8 & 5.5 \\
\hline & Fig $23(7)$ & $1262 \mathrm{C} 10-4,131-132$ & 4.9 & 4.8 & 5.7 & 1.3 & 3.8 & 5.1 \\
\hline \multirow[t]{3}{*}{ Fasciculithus sp. A } & Fig $25(1)$ & $1262 \mathrm{C} 10-3,74-75$ & 5.4 & 6.7 & 7.2 & 1.7 & 5.4 & 7.1 \\
\hline & Fig $25(2)$ & $1262 \mathrm{C} 10-3,74-75$ & 4.5 & 5.2 & 5.8 & 1.0 & 4.9 & 5.9 \\
\hline & Fig $25(3)$ & $1262 \mathrm{C} 10-3,74-75$ & 3.9 & 4.9 & 5.3 & 0.9 & 4.0 & 4.8 \\
\hline \multirow[t]{3}{*}{ F. realeae } & Fig $25(5)$ & $1209 \mathrm{C}-14-1,56-67$ & 4.6 & 6.4 & 6.4 & 1.4 & 4.7 & 6.0 \\
\hline & Fig $25(6)$ & $1209 \mathrm{C}-14-1,56-67$ & 4.5 & 5.9 & 6.1 & 1.0 & 4.5 & 5.5 \\
\hline & Fig $25(7)$ & $1209 \mathrm{C}-14-1,56-67$ & 5.0 & 6.0 & 6.7 & 0.9 & 5.7 & 5.9 \\
\hline \multirow[t]{5}{*}{ F. vertebratoides } & Fig $26(1)$ & 356Z 23R-1W, 70-71 & 5.2 & 5.4 & 6.9 & 1.5 & 3.6 & 6.7 \\
\hline & Fig $26(2)$ & 356Z 23R-1W, 70-71 & 4.6 & 5.8 & 6.3 & 1.0 & 3.6 & 5.7 \\
\hline & Fig $26(3)$ & $1262 \mathrm{C} 10-6,23-24$ & 4.6 & 7.6 & 7.9 & 2.0 & 4.6 & 6.6 \\
\hline & Fig $26(4)$ & $11937-4,40-41$ & 4.8 & 6.0 & 6.4 & 1.3 & 3.5 & 6.1 \\
\hline & Fig $26(5)$ & $356 \mathrm{Z} 23 \mathrm{R}-1 \mathrm{~W}, 70-71$ & 4.3 & 5.8 & 5.8 & 1.0 & 3.0 & 5.3 \\
\hline
\end{tabular}




\section{References:}

Aubry, M.-P., Rodriguez, O., Bord, D., Godfrey, L., Schmitz, B and Knox, R.W. O’B.: The first radiation of fasciculiths: morphologic adaptations of the coccolithophores to oligotrophy, Austrian J. Earth Sci, 105, 29-38, (2012).

Bralower, T.J.: Data report: Paleocene-early Oligocene calcareous nannofossil biostratigraphy, ODP Leg 198 Sites 1209 , 1210, and 1211 (Shatsky Rise, Pacific Ocean), in: Proceedings ODP, Scientific Results, 198, edited by: Bralower, T.J., Premoli Silva, I., and Malone, M.J., College Station, Texas (Ocean Drilling Program), 1-15, doi:10.2973 /odp.proc.sr.198.115, 2005. Galeotti, S., Moretti, M., Cappelli, C., Phillips, J., Lanci, L., Littler, K., Monechi, S., Petrizzo M.R., Premoli Silva, I., Zachos J. C.: The Bottaccione section at Gubbio, central Italy: a classical Paleocene Tethyan setting revisited, Newsl Stratigr, 48,325339, DOI: $10.1127 / \mathrm{nos} / 2015 / 0067$

Monechi, S., Reale, V., Bernaola, G. and Balestra, B.: The Danian/Selandian boundary at Site 1262 (South Atlantic) and in the Tethyan region: Biomagnetostratigraphy, evolutionary trends in fasciculiths and environmental effects of the Latest Danian Event, Mar. Micropaleontol, 98, 28-40, https://doi.org/10.1016/j.marmicro.2012.11.002, 2013.

Perch-Nielsen, K.: Einige neue Coccolithen aus dem Paleozän der Bucht von Biskaya, Bulletin of the Geological Society of Denmark, 2,347-361, 1971.

Perch-Nielsen, K.: Albian to Pleistocene calcareous nannofossils from the western South Atlantic, DSDP Leg 39, in: Initial Reports of the Deep Sea Drilling Project, edited by: Supko, P.R., Perch-Nielsen, K., et al., U.S. Government Printing Office, Washington, 39: 699-823. https://doi.org/10.2973/dsdp.proc.39.131.1977,1977.

Zachos, J.C., Kroon, D., Blum, P., et al.,: Shipboard Scientific Party, 2004. Leg 208 summary, in: Proceedings of the Ocean Drilling Program, Initial Reports 208, edited by Zachos, J.C., Kroon, D., Blum, P., et al., College Station TX (Ocean Drilling Program), 1-112, doi:10.2973/odp.proc.ir.208.108, 2004. 